\title{
The Influence of Meta-Experimental Factors on Compliance and Attitudes: Participant Motivation and Experimenter Demeanor
}

\author{
Kelly Jo Hubers, Elizabeth R. Graf, and Sherri B. Lantinga \\ Department of Psychology \\ Dordt College \\ 498 4th Ave NE \\ Sioux Center, lowa 51250 USA
}

Received:February 5, 2003 Accepted: April 25, 2003

\begin{abstract}
An experimental study examined the effects of participant motivation (required vs. voluntary participation) and experimenter demeanor (participants treated as co-researchers or as dataproducers) on compliance and participant attitude. Participants completed a meaningless task and then filled out a questionnaire about their attitudes toward the experimenter and task. Compliance was measured by how long and how accurately participants worked on the task. Results indicate that Participant Motivation type affects attitudes toward the experimenter and task and Experimenter Demeanor affects compliance on a task. Voluntary Co-Researcher participants had more positive attitudes and marked on the most number of pages but did not complete the task correctly compared to other participants. Future research could increase the impossibility of the task to see how much participants will comply, and should look at the effect on compliance that a greater difference in position between experimenters and participants may have.
\end{abstract}

\section{INTRODUCTION}

Psychological research is conducted to study human behavior, but behavior is influenced by factors in the experimental setting itself. One factor that affects behavior is the motivation of the participants for being in the study. A psychology experiment cannot be conducted without participants, and in today's world, most people are too busy to serve as volunteers. Monetary inducements are sometimes offered but even this may fail to recruit needed participants.

Participants may have different motivations for being in an experiment and they may have preconceived attitudes about research in general [1, 2]. Many universities require introductory psychology students either to participate in a research experiment or write a 5-10 page research paper [3].
The American Psychological Association requires that participation be voluntary, or if required, that an equal alternative to the study is provided [4]. Rosenthal argues that requiring students to participate borders on coercion. Researchers may think that participants are in full cooperation when in fact they may feel that they are forced to be part of the study [5]. Maughan and Higbee had participants estimate the effects of incentives for participation on compliance and obedience in well-known studies [6]. Students thought that required or paid participants would comply more than voluntary participants [6]. Introductory psychology students are a large part of the subject pool used in psychological research [3]. Participant motivation (required or voluntary) can affect the attitudes of the participants about the study and consequently their actions. 
Because some participants are required to be in a study, they may feel that they are required to provide the "correct" information in order to get course credit [7]. This could be another variable influencing participant's behavior other than the manipulations of the experimenter. Furthermore, if participants believe they are involved in a meaningful task, they are more likely to comply than participants who believe their task is useless $[2,8]$.

Orne looked at how perception of meaning of a task affected the way participants complied when asked to complete the task [9]. For example, participants were instructed to tear up each completed sheet of math problems before completing the next one in a pile. Five and one half hours later, the experimenter stopped the participants. The participants reported that they worked so long because they believed the experiment could be testing something such as endurance that they had not been told about [9]. Orne found a direct relationship between how participants received information on the study and how they responded to the instructions given by the experimenter. In his experiment, participants instructed by a cold and clinical experimenter reacted differently compared to participants instructed by a casual and friendly experimenter $[9,10]$.

The way information is presented in an experiment can elicit a certain response from the participants [2]. In his famous study, Milgram showed that participants will comply with the instructions given by an authority figure to the point of physically harming or even killing other humans [11]. These participants may be responding to the presentation of the instructions rather than the instructions themselves. Orne demonstrates this on a smaller scale through his experiment on the way information is presented to participants [10]. Information presented in a relaxed manner led to increased compliance among participants, while information presented in a threatening way decreased compliance [10]. These studies independently examined the relationships between how participants were treated and how they behaved during the study, and between motivation for participation and behavior during the study. The current study examines the interaction of how participants were treated and their motivation for participation on their behavior. We predicted that voluntary participants who were treated more collegially would work on a task longer and have more positive attitudes toward the experimenters and the task than would participants treated distantly or who were required to participate.

\section{METHOD}

\section{a. Design and Operationalization of Variables}

A 2 (Participant Motivation: Required, Voluntary) x 2 (Experimenter Demeanor: "Data-Producers," "Co-Researchers") between-subjects factorial design was used [3]. Six sessions were randomly assigned to the Experimenter Demeanor conditions.

Bechtold's distinction between DataProducers and Co-Researchers was utilized [3]. Data-Producer participants were treated as a means of acquiring information. They were given minimal respect as a participant and not valued as people deserving real choices who will learn from their participation [3]. The experimenters did not talk to the participants or discuss the experiment until the consent form was distributed. The consent form contained formal language and little information about the experiment. Experimenters demonstrated a formal politeness, but conveyed the impression that the data-producers were less important than the experimenters themselves. Co-Researcher participants were viewed as colleagues and treated with genuine interest and equality. Experimenters showed concern that the participants would learn from their experience [3]. Experimenters mingled with participants on arrival to put them at ease. Experimenters established a collaborative environment with the participants by speaking in a friendly, informal fashion. Experimenters smiled more and made conversation to indicate a warmer setting than in the Data-Producer condition. The consent form was consistent with the friendly manner of the experimenters.

\section{b. Participants}

\section{Seventy Dordt College introductory}


psychology students (41 female, 29 male) participated in the study. Participants were from three different introductory psychology classes. One class was randomly assigned to the Required condition; thirty-six students participated. Thirty-four Voluntary participants received extra credit for participation. All students were allowed to sign up for a time slot; the time slots were then randomly assigned to one of the two levels of the Experimenter Demeanor condition. Participants ranged in age from 17-28, and all were Caucasian.

\section{c. Materials}

A questionnaire measuring suspiciousness of the experiment was adopted from Kooiman and Van Wyk [12]. Attitudes toward the experimenters and the task were measured by 14 items on 5 -point Likert-type scales, anchored with opposing statements (e.g. "I thought the task was worthwhile" vs. "I thought the task was a waste of time"; "I would like to get to know her better as a person" vs. "I wouldn't want anything else to do with the experimenter"); higher numbers reflected more negative attitudes. Self-reported general compliance of the participants was measured with five items on 5-point Likert-type scales anchored with opposing statements (e.g., "I avoid doing favors for others" vs. "I'm willing to do favors for others"); higher numbers indicated more compliance. The Marlowe-Crowne Social Desirability Scale, of 33 true/false items, measured how compelled participants felt to follow social norms; higher numbers meant more social desirability [13]. Marlowe and Crown reported an internal reliability alpha coefficient of .88 for their Social Desirability Scale. Five demographic items, two items referring to participation in previous experiments, and two manipulation checks were included. One, a 5-point Likerttype scale, assessed the degree to which participants felt like a Co-Researcher or Data-Producer, and the other, a fixed alternative, asked if participation was Required or Voluntary.

\section{d. Procedure}

Participants were told they would be participating in a study of spontaneous creativity. Participants arrived and were treated according to their Data-Producer or Co-Researcher conditions. Participants were seated in study cubicles and consent forms were distributed, read and signed. DataProducer participants received a formal version of the consent form with minimal information. Opportunity was given to ask questions but the experimenter's curt demeanor discouraged any questions. CoResearcher participants received a consent form written in a friendlier tone. The experimenter's amiable demeanor encouraged questions and discussion if anything was unclear to the participants.

Written instructions, 20 sheets of 8.5 inch $x$ 11-inch graph paper (6x6 squares per inch) per participant, and pencils were handed out. Instructions read:

Remain silent at all times during the rest of the experiment. You have 20 sheets of graph paper and a pencil. Color each box individually in varying shades beginning with the box in the top left corner and proceeding across the row from left to right. Complete each row before beginning the next one. Touching boxes in any direction (left, right, top, or bottom) cannot be the same shade. Color each sheet completely before moving on to the next sheet. When you have finished, put all your materials back in the folder and take it to room 012 to complete a questionnaire. The tasks of each person will take different lengths of time. Do not feel rushed. Please remain completely silent at all times during the experiment.

The task and instructions were standard across all conditions and silence was maintained at all times. The study cubicles were used to diminish social comparisons. Those still working on the task were stopped after $50 \mathrm{~min}$. The time each participant worked on the task, the number of boxes they shaded correctly, and the number of pages marked was recorded. Participants then filled out the questionnaire.

During the debriefing, participants learned that creativity was used as a cover story so they would not focus on their compliance. After all the conditions were run, participants in the required condition were informed that they would receive the same amount of extra credit as the voluntary participants. 


\section{RESULTS}

One participant from the Required Data-Producer condition informed the experimenter during the debriefing that he lied on his questionnaire; therefore, his questionnaire results were dropped. Three participants shaded over 2200 boxes, which was more than 3 standard deviations away from the mean, so these data were dropped as outliers. The manipulation checks indicated that $97 \%$ of participants correctly reported their Participant Motivation condition (Required, Voluntary). A t-test indicated no significant difference between conditions on perception of Experimenter Demeanor $(M=3.57, S D=1.00), t(68)=$ $0.62, p=.54$.

\section{a. Measures of the Dependent Variables}

Compliance was measured in 5 ways: number of minutes spent on the task (range 1-50), number of boxes shaded correctly (range $0-2200$ ), number of pages marked on during the task (range 0-20), participant attitudes toward the experimenter and task, and self-reported general compliance. Boxes were correct if there was an attempt to shade individual boxes rather than scribbling on the entire sheet. Some participants marked on no pages while others marked in some way on all 20 pages. Questionnaire items were averaged to derive attitude and compliance scores. Table 1 displays the means and standard deviations for the dependent variables overall and by condition. The mean attitude score toward the experimenters and the task fell at the midpoint. The mean score for general compliance suggested participants tend to think of themselves as compliant. We predicted that Voluntary participants who were treated collegially would work on a task longer and have more positive attitudes toward the experimenters and the task than

Dependent Variables

\begin{tabular}{|c|c|c|c|c|c|c|c|c|c|c|}
\hline \multirow[b]{2}{*}{ Condition } & \multicolumn{2}{|c|}{$\begin{array}{c}\text { Number of } \\
\text { Minutes }\end{array}$} & \multicolumn{2}{|c|}{$\begin{array}{c}\text { Number of } \\
\text { Boxes }\end{array}$} & \multicolumn{2}{|c|}{$\begin{array}{c}\text { Number of } \\
\text { Pages }\end{array}$} & \multicolumn{2}{|c|}{$\begin{array}{c}\text { Participant } \\
\text { Attitudes }\end{array}$} & \multicolumn{2}{|c|}{$\begin{array}{c}\text { General } \\
\text { Compliance }\end{array}$} \\
\hline & Mean & SD & Mean & SD & Mean & SD & Mean & SD & Mean & SD \\
\hline Overall & 34.90 & 17.11 & 308.67 & 321.52 & 4.57 & 7.07 & 2.98 & 0.60 & 3.44 & 0.65 \\
\hline $\begin{array}{l}\text { Required Co- } \\
\text { Researchers }\end{array}$ & 33.60 & 17.13 & 370.62 & 246.62 & 4.93 & 7.65 & 3.26 & 0.78 & 3.40 & 0.74 \\
\hline $\begin{array}{l}\text { Voluntary } \\
\text { Co- } \\
\text { Researchers } \\
\end{array}$ & 26.00 & 15.80 & 161.71 & 265.27 & 9.50 & 9.38 & 2.73 & 0.42 & 3.46 & 0.69 \\
\hline $\begin{array}{l}\text { Required } \\
\text { Data } \\
\text { Producers }\end{array}$ & 39.95 & 15.32 & 217.00 & 232.08 & 2.43 & 4.21 & 3.04 & 0.52 & 3.25 & 0.66 \\
\hline $\begin{array}{l}\text { Voluntary } \\
\text { Data } \\
\text { Producers }\end{array}$ & 36.80 & 18.37 & 461.42 & 408.61 & 3.10 & 5.85 & 2.90 & 0.56 & 3.67 & 0.48 \\
\hline
\end{tabular}

Table 1. Means and Standard Deviations (SD) by Treatment Condition on Dependent Variables 
would participants treated distantly or who were required to participate. The alpha level was set at .05. A factorial analysis of variance (ANOVA) on the number of minutes spent on the task showed a significant effect of Experimenter Demeanor, $F(1,66)=4.29, p=.04$ (see Figure 1). Data-Producer participants spent more minutes working on the task than did CoResearcher participants. The ANOVA showed no significant effect for Participant Motivation, $F(1,66)=1.63, p=.21$, or for the interaction between Experimenter Demeanor and Participant Motivation, $F(1$, $66)=0.34, p=.56$.

An ANOVA on the number of boxes shaded correctly showed a marginally significant effect for the interaction between Experimenter Demeanor and Participant Motivation, $F(1,65)=4.31, p=.05$ (see Figure 2). Post-hoc Tukey tests showed that in the Voluntary participation condition, Data-Producers $(M=559.40, S D=591.76)$ shaded significantly more boxes correctly than Co-Researchers $(M=161.71, S D=$ 265.27), $F(1,60)=14.04, p<.05$. In the Required participation condition, there was no significant difference on the number of boxes shaded between Data-Producers and Co-Researchers $F(1,60)=3.19$, failed to reach significance at $p<.05$. There were no significant main effects for Experimenter Demeanor or Participant Motivation on the number of boxes shaded correctly, all Fs (1, $65)<1.67, p s>.20$.

Experimenter Demeanor had a significant effect on the number of pages marked, $F(1,66)=7.30, p=.01$ (see Figure $3)$. The Co-Researcher participants marked on more pages than the Data-Producer participants. There was no significant effect for Participant Motivation on the number of pages marked, $F(1,66)=2.74, p=.10$, or for the interaction between Experimenter Demeanor and Participant Motivation, $F(1$, 66) $=1.31, p=.26$.

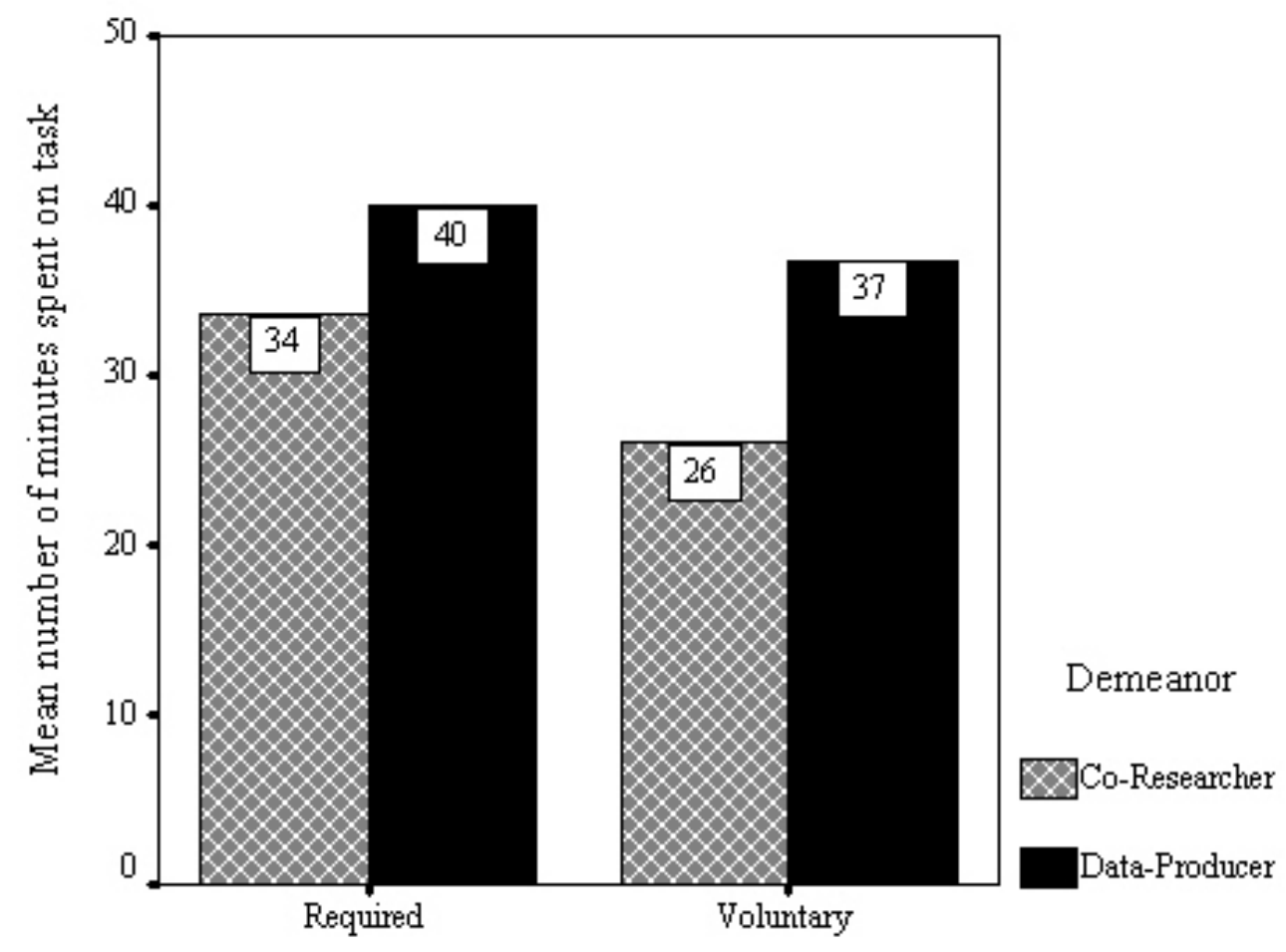

\section{Participant Motivation}

Figure 1. The effects of Experimenter Demeanor and Participant Motivation on the mean number of minutes spent on the task. 


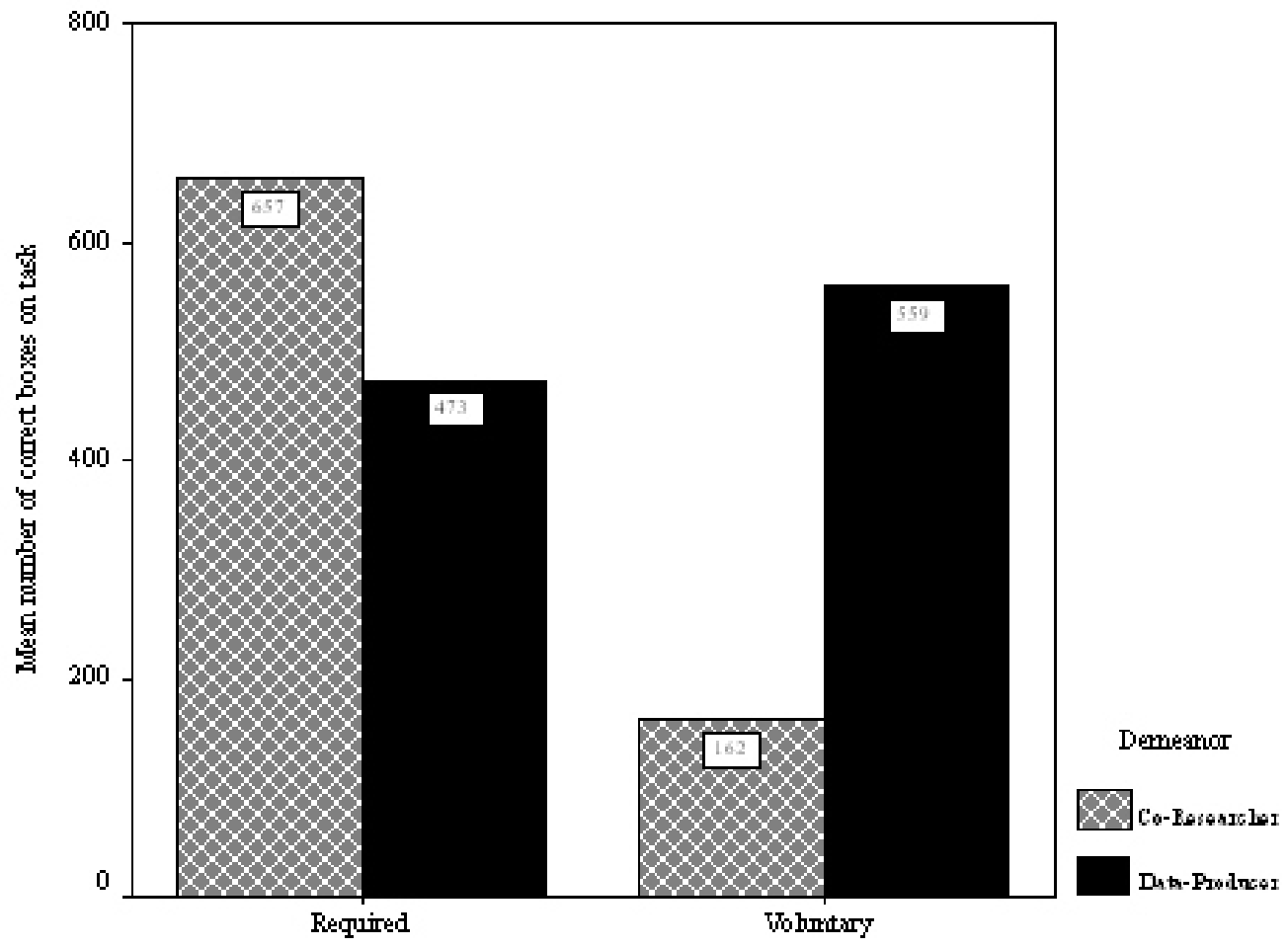

Participant Motivation

Figure 2. The effects of the interaction between Experimenter Demeanor and Participant Motivation on the number of boxes shaded correctly.

Another ANOVA showed a significant effect for Participant Motivation on attitudes, $F(1,60)=4.54, p=.04$ (see Figure 4). Voluntary participants had significantly more positive attitudes toward the experimenter and the task than did Required participants. Lower numbers indicate more positive attitudes. There was no significant effect for Experimenter Demeanor or for the interaction between Experimenter Demeanor and Participant Motivation on the overall attitude score, all Fs $(1,60)<1.97$, ps $>.17$.

\section{a. Exploratory Analyses}

Other variables that might have affected compliance were also examined. Gender had no significant effects on any measures of the dependent variable, either as main effects or interactions, all $F s<3.08$, ps $>.08$. Social desirability score and attitude were negatively related, $r(67)=-$ $.26, p=.04$. Internal reliability was somewhat lower than reported in the literature (.88); our alpha coefficient was .79. Social desirability decreased as the participants' attitudes toward the experimenter and the task became more negative. Number of minutes spent on a task and the number of boxes shaded correctly were positively related, $r(70)=.37$, $p=.00$. Number of pages marked on during the task and number of boxes shaded correctly were negatively related, $r(70)=$ $.31, p=.01$. Not surprisingly, more boxes were shaded the longer the participants spent on the task; however, the more pages participants marked on, the less boxes were shaded correctly. Age and self-reported general compliance were unrelated to other measures, all $r s<.18, p s>.14$. 
In sum, these results partially supported the hypothesis in that Voluntary participants reported more positive attitudes toward the experimenters and the task than did Required participants. Also, CoResearchers marked on more pages than Data-Producers, but Data-Producers worked on the task longer. However, the interaction between Experimenter Demeanor and Participant Motivation was not consistent with our prediction, because Voluntary CoResearcher participants shaded the least number of boxes correctly and Voluntary Data-Producer participants shaded correctly the most number of boxes.

\section{DISCUSSION}

The purpose of this study was to determine how participant motivation and the experimenter's attitude toward participants would affect compliance and attitudes. We hypothesized that Voluntary Co-Researchers would work on a task longer and have more positive attitudes toward the experimenters and the task than would Data-Producers and Required participants. Results showed Voluntary participants did have more positive attitudes than Required participants, but Experimenter Demeanor did not have an influence on attitudes. Thus, the attitudes toward the experimenter and the task may have been formed before the participant ever reached the experiment and were not significantly affected by the experimenter's actions. Data-Producers worked longer on the task than did Co-Researchers, suggesting that they felt obligated to do as the experimenters instructed. Co-Researchers marked on more pages, suggesting that they made an attempt to finish the task by marking all 20 pages. Another interpretation is that Co-Researchers were attempting at a covert rebellion similar to Milgram's subterfuges [11]. Participants might have felt compelled to complete the experiment but at the same time tried to undermine it by not performing the task correctly.



Participant Motivation

Figure 3. The effects of Experimenter Demeanor and Participant Motivation on the mean number of pages marked. 


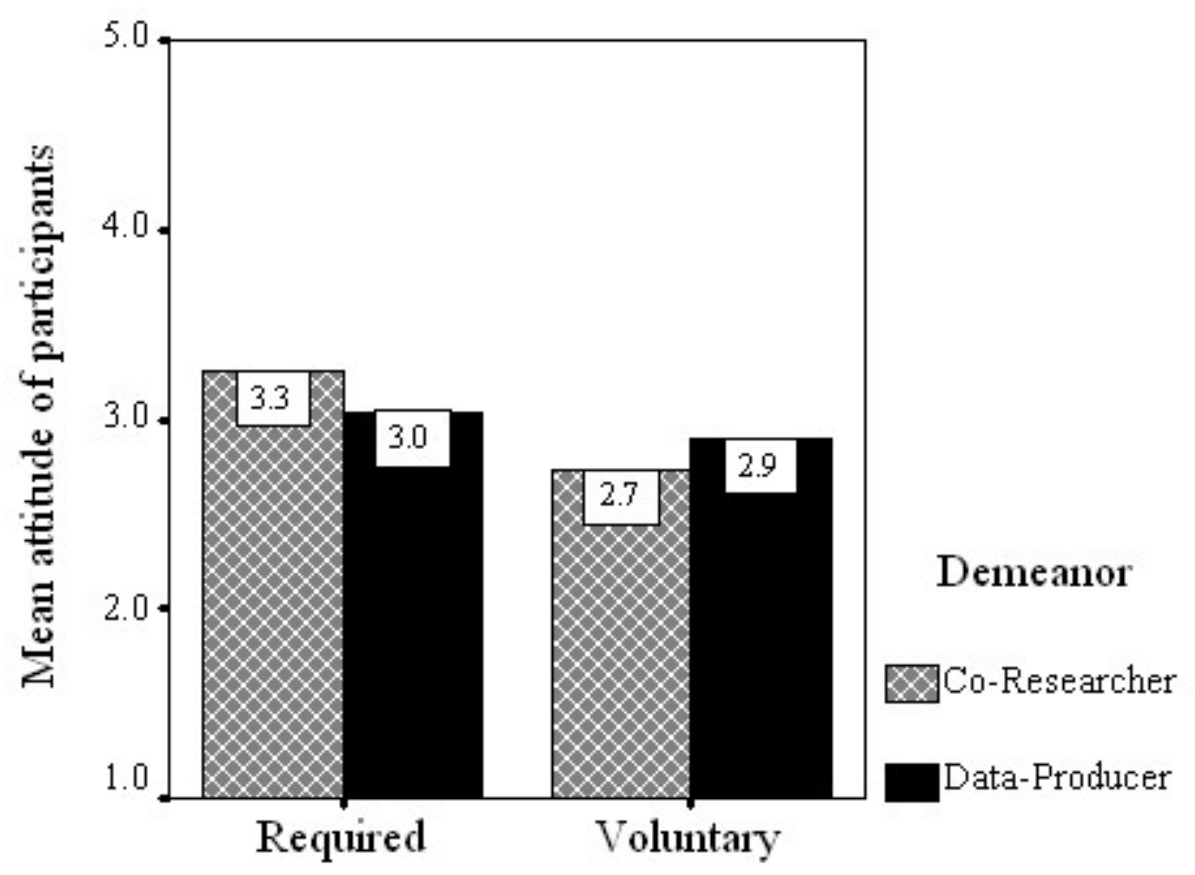

Participant Motivation

Figure 4. The effects of Experimenter Demeanor and Participant Motivation on the mean attitudes of participants toward the experimenter and the task. Higher numbers indicate more negative attitudes.

Our results also showed a significant interaction between Experimenter Demeanor and Participant Motivation on the number of boxes shaded correctly. Voluntary Data-Producer participants shaded the most boxes suggesting that participants who were treated distantly, but were voluntary, made the most effort to complete the task accurately. These participants may have tried to justify their participation in the study - they volunteered to come to the study but received no external reward from the experimenters; therefore, performing the task correctly may have been a means to make their participation worthwhile.

Our research showed that how the experimenter treated the participants did affect the number of minutes spent on a task as well as on the number of pages marked during the task. This suggests that the way information is presented does affect the way participants behave. This direct relationship between how participants received information and consequent behavior in the experiment is supported by Orne [1] and Adair [2]. This relates to Bechtold's premise that experimenters should be careful to treat participants as co-researchers instead of reducing them to sources of data [3]. We did not find any difference in compliance between Voluntary and Required participants. Maughan and Higbee found that students did not estimate a difference in compliance based on incentive for participation [6]. This study found that compliance to the task was not affected by gender. This is consistent with studies finding that male and females conform similarly when responses are private [14], as in this study.

Time and resources limited this study. Each Data-Producer and CoResearcher condition had both Voluntary and Required participants and it is possible that a group mentality influenced the actions of individual participants during the study. Participants completed their tasks in 
cubicles; however, experimenters often observed participants craning their necks to see how others were working on their tasks. If there had been more time and resources to run participants in separate rooms, they would not have known when other participants finished and their own decisions to leave would not have been influenced by others.

The study was also limited in that all participants seemed to feel like a source of data. Participants rated their perceived treatment on a 5-point Likert-type scale (1 = "I feel that the experimenter regarded me not as a person but as a source of data." Or $5=$ "I feel the experimenter was interested in me as a person"). Our manipulation check showed participants felt more like a source of data regardless of condition. No participant reported feeling like a CoResearcher, but more of the participants in the Co-Researcher condition reported positive attitudes toward the experimenter and task. Thus, the experimenter's definition of Co-Researcher and DataProducer appeared to differ from the definitions of participants.

Future research, if replicating this study, could increase the impossibility of completing the task. Participants were given 20 pages of graph paper; some participants wrote on all 20 pages and left the experiment. Increasing the number of pages may make the task seem more impossible to complete.

Also, in this study both the experimenters and the participants were undergraduate students. It is possible that a greater difference of position between experimenters and participants might elicit more respect from undergraduate participants. For example if the experimenter was a graduate student or a professional researcher and perceived as an authority figure, undergraduate participants might have increased compliance.

In conclusion, these findings are applicable to all research using human participants. In particular, this study showed that participant responses in an experimental setting are significantly influenced by an interaction between why they are there and how they are treated. Researchers need to consider the possible implications when conducting experiments, interpreting results, and generalizing results outside of the experimental setting.

\section{ACKNOWLEDGEMENTS}

An earlier version of this paper was presented at the Tenth Midwest Institute for Students and Teachers of Psychology in Glyn Ellen, Illinois, on February 28 - March 1,2003 . We thank the two anonymous reviewers whose helpful suggestions significantly strengthened this paper.

\section{REFERENCES}

1. Adair, J. G. (1972). Demand characteristics or conformity?: Suspiciousness of deception and experimenter bias in conformity research. Canadian Journal of Behavioral Science, 4, 238-248.

2. Adair, J. G. (1973). The human subject: The social psychology of the psychological experiment. Boston: Little, Brown and Company.

3. Bechtold, J. I. (1996, July). The participant as colleague vs. the subject as data-producer: The ethics and practice of human experimentation with "voluntary" introduction to psychology students: A Christian approach. Paper presented at the meeting of the ASA and CSCA 51st Annual Meeting, Toronto, Ontario.

4. American Psychological Association. (1992). Ethical principles of psychologists and code of conduct. American Psychologist, 47, 1597-1611.

5. Rosenthal, R. (1994). Science and ethics in conducting, analyzing, and reporting psychological research. Psychological Science, 5, 127-134.

6. Maughan, M. R. C., \& Higbee, K. L. (1981). Effect of subjects' incentives for participation on estimated compliance for self and others. Psychological Reports, 49, 119-122.

7. Fisher, C. B., \& Fyrberg, D. (1994). Participant partners college students weigh the costs and benefits of deceptive research. American Psychologist, 49, 417-427.

8. Myers, A., \& Hansen, C. H. (1997). Experimental psychology (4th ed.). Pacific Grove, CA: Brooks/Cole Publishing Company. 
9. Orne, M. T. (1962). On the social psychology of the psychological experiment: With particular reference to demand characteristics and their implications. American Psychologist, 17, 776-783.

10. Orne, M. T. (1964). The contribution of nondeprivation factors in the production of sensory deprivation effects: The psychology of the "panic button." Journal of Abnormal and Social Psychology, 68, 3-12.

11. Milgram, S. (1969). Obedience to authority. New York: Harper \& Row, Publishers.
12. Kooiman, H. F., \& Van Wyk, C. A. (2001). Effects of chair comfort and arrangement on experiment-related suspiciousness. Unpublished manuscript, Dordt College.

13. Marlowe, D., \& Crowne, D. P. (1964). The approval motive. New York: John Wiley \& Sons, Inc.

14. Eagly, A. H., Wood, W., \& Fishbaugh, L. (1981). Sex differences in conformity: Surveillance by the group as a determinant of male nonconformity. Journal of Personality and Social Psychology, 40, 384-394.

\section{Support Undergraduate Research}

\section{Subscribe to the American Journal of Undergraduate Research}



\author{
Individual Rate: \$20/year \\ Institutional Rate: \$40/year
}

Back Issues at \$5/issue

Write to: AJUR Subscriptions

Physics 205

University of Northern lowa

Cedar Falls, IA 50614-0150 Cheques payable to "AJUR"

\section{A REFEREED JOURNAL FOR UNDERGRADUATE RESEARCH} IN THE PURE \& APPLIED SCIENCES, MATHEMATICS, ENGINEERING, TECHNOLOGY, AND RELATED AREAS IN EDUCATION 